SU-4240-719

hep-ph/0004105

\title{
Complementary Ansatz for the neutrino mass matrix
}

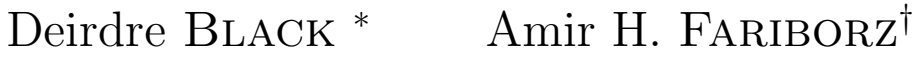 \\ Salah NAsRI开 Joseph Schechter 卢 \\ Department of Physics, Syracuse University, Syracuse, NY 13244-1130, \\ $U S A$.
}

\begin{abstract}
We propose a simple Ansatz for the three generation neutrino mass matrix $M_{\nu}$ which is motivated from an $\mathrm{SO}(10)$ grand unified theory. The Ansatz can be combined with information from neutrino oscillation experiments and bounds on neutrinoless double beta decay to determine the neutrino masses themselves and to reconstruct, with some assumptions, the matrix $M_{\nu}$.
\end{abstract}

*Electronic address: black@physics.syr.edu

$\dagger$ Electronic address: amir@suhep.phy.syr.edu

$\ddagger$ Electronic address : snasri@suhep.phy.syr.edu

$\S$ Electronic address : schechte@suhep.phy.syr.edu 


\section{INTRODUCTION}

In the last few years there has been another wave of excitement regarding the question of neutrino masses. This is largely due to the many new experiments testing neutrino oscillations, most notably the positive indications obtained by Super Kamiokande on atmospheric neutrino oscillations [1]. Similar indications come from other experiments [2 [5]. The solar neutrino experiments have for many years provided independent evidence for neutrino oscillations [6 10]. Accelerator and reactor experiments have also played an important role. They have furnished strict bounds on neutrino oscillation parameters [11 [16]. In the case of the LSND experiment [16] at Los Alamos evidence for $\bar{\nu}_{\mu} \rightarrow \bar{\nu}_{e}$ oscillation has been reported. See refs [17 for recent reviews.

It is hoped that new experimental results can be used to determine the neutrino squared mass differences and mixing angles. In turn, these may help to infer the neutrino mass matrix. This is presumably a possible gateway to a more fundamental theory beyond the standard model. Of course this is a highly speculative area, and even though there are many imaginative proposals [18], it seems fair to say that the the true answer is essentially unknown. In order to make progress in this direction, it seems useful to investigate various plausible Ansatze for the neutrino mass matrix. From this point of view we propose the Ansatz for the 3 generation neutrino mass matrix, $M_{\nu}$ :

$$
\operatorname{Tr}\left(M_{\nu}\right)=0
$$

and investigate its consequences. We are considering the neutrinos to be represented by 2-component spinors so that, in the most general situation, $M_{\nu}$ is an arbitrary symmetric complex matrix.

As we will see in section II, Eq. (1.1) can be motivated from an $\mathrm{SO}(10)$ grand unified model [19], in which it may be derived with some assumptions. Physically, Eq. (1.1) corresponds to the well known approximate signature of grand unification that $\frac{m(b)}{m(\tau)} \simeq 3$. Furthermore we will see in sections IV and V that Eq. (1.1) can be straightforwardly combined with experimental information to get an idea of the neutrino masses themselves as well as the "texture" of $M_{\nu}$. Relevant matters of notation are discussed in section III while a summary is presented in section VI.

\section{PLAUSIBILITY OF THE ANSATZ}

In the $\mathrm{SO}(10)$ grand unification model each generation contains one light massive two component neutrino and also a very heavy one which is "integrated out" according to the 
"seesaw mechanism" [20]. The effective $3 \times 3$ neutrino mass matrix takes the form:

$$
M_{\nu}=M_{L}-M_{D}^{T} M_{H} M_{D}
$$

where $M_{L}, M_{H}$ and $M_{D}$ are respectively the mass matrices of the light neutrinos, heavy neutrinos and heavy-light mixing (or "Dirac matrix"). Generally the second, seesaw, term is considered to dominate. Here however we shall assume the first term to be the dominant one. This is necessary for the present derivation of Eq. (1.1) to hold. Also, a rough order of magnitude estimate for the second term would be $\frac{m(\tau)^{2}}{10^{16} \mathrm{GeV}}$ or about $3 \times 10^{-7} \mathrm{eV}$. Thus, the seesaw term could be negligible if neutrino masses turn out to be appreciably larger than this value. Now in SO(10), Higgs mesons belonging to the 10, 120 and 126 representations can contribute to the fermion masses at tree level. One has [21] for the down quark, charged lepton and light neutrino mass matrices,

$$
\begin{aligned}
M_{d} & =a S(10)+b A(120)-\frac{1}{3} c S(126) \\
r M_{e} & =a S(10)+d A(120)+c S(126) \\
s M_{L} & =e S(126)
\end{aligned}
$$

where $a, b, c, d, e$ are numbers representing Higgs meson vacuum values. S(10), A(120) and $\mathrm{S}(126)$ are the matrices of the Yukawa type constants which couple the fermions to the 10, 120 and 126 Higgs mesons respectively; the matrices S(10) and S(126) must be symmetric while $\mathrm{A}(120)$ is antisymmetric. Finally, $r \simeq 3$ is a renormalization factor for comparing the quark masses with the charged lepton masses at a low energy scale rather than at the grand unified scale; $s$ is a similar factor for the neutrino masses. With the stated assumption that the $M_{L}$ term dominates in Eq. (2.1) we get

$$
\operatorname{Tr}\left(M_{\nu}\right) \propto \operatorname{Tr}\left(M_{d}\right)-r \operatorname{Tr}\left(M_{e}\right)
$$

which clearly also holds when any number of 10's or 120's are present but only a single 126 . The matrices appearing in Eq. (2.3) are so far essentially unrestricted complex ones. To proceed, we make the further assumption that the matrices are hermitian. Then $M_{d}$ and $M_{u}$ may each be brought to diagonal form by unitary transformations. Thus the right hand side of Eq. (2.3) may be evaluated to yield approximately,

$$
\operatorname{Tr}\left(M_{\nu}\right) \propto m(b)-r m(\tau) \simeq 0
$$

according to a well known numerical success, based on the observation that $r \simeq 3$, of grand unification [22]. Note that we have not needed to assume that the mass matrix has any 
zero elements. f $^{2}$ Even if the cancellation on the right hand side of Eq. (2.4) is not perfect, it should still be a good approximation. In an $\mathrm{SO}(10)$ model where the mass matrices are hermitian, $M_{\nu}$ will be real symmetric. We will investigate this case and also the possibility that the more general case holds.

\section{SOME NOTATION}

Our plan is to combine the Ansatz Eq. (1.1) with experimentally obtained results on neutrino oscillations in order to learn more about $M_{\nu}$ itself. For this purpose it may be helpful to set down our notation [23] for the pieces of the effective $\mathrm{SU}(2)_{L} \times \mathrm{U}(1)$ theory involving neutrinos and to make some related remarks.

The free Lagrangian containing three two component massive fields is:

$$
\mathcal{L}_{\text {free }}=-i \rho^{\dagger} \sigma_{\mu} \partial_{\mu} \rho-\frac{1}{2}\left(\rho^{T} \sigma_{2} M_{\nu} \rho+\text { h.c. }\right)
$$

where $M_{\nu}=M_{\nu}^{T}$ is the (not yet diagonalized) neutrino mass matrix of the underlying theory to be identified with the matrix in Eq. (1.1). Note that we are free to multiply the first mass term in Eq. (3.1) by an overall arbitrary phase which is a matter of convention. It is possible 23] to find a unitary matrix $U$ which brings $M_{\nu}$ to real, positive, diagonal form in the following way:

$$
U^{T} M_{\nu} U=\hat{M}, \quad \hat{M}=\operatorname{diag}\left(m_{1}, m_{2}, m_{3}\right) .
$$

The mass diagonal fields $\nu$ are then

$$
\rho=U \nu
$$

Similarly, the column vector of left handed negatively charged leptons in the underlying theory, $E_{L}$ is related to the mass diagonal fields $e_{L}$ by

$$
E_{L}=\Omega e_{L},
$$

where $\Omega^{\dagger}=\Omega^{-1}$.

\footnotetext{
*In [24] a similar mechanism was studied for $M_{H}$ where, in addition, a special combined FritzschStech Ansatz was used. Here we are not making any special Ansatz of this type for the mass matrices.
} 
Combining factors from Eq. (3.3) and Eq. (3.4) we obtain the unitary mixing matrix, $K$ for the charged current weak interaction,

$$
K=\Omega^{\dagger} U
$$

This appears in the Lagrangian term,

$$
\mathcal{L}_{\text {int }}=\frac{i g}{\sqrt{2}} W_{\mu}^{-} \bar{e}_{L} \gamma_{\mu} K \nu+\text { h.c. }
$$

where a conventional four component Dirac notation with $\gamma_{5}$ diagonal is being employed and $\nu$ has only the first two components non zero. Next we parameterize $K$ [23]. It is possible to restrict $\operatorname{det} K=1$ by adjusting an overall phase which can be absorbed in $\bar{e}_{L}$. Then we write

$$
K=\omega_{0}(\alpha) \omega_{23}\left(\eta_{23}, \phi_{23}\right) \omega_{12}\left(\eta_{12}, \phi_{12}\right) \omega_{13}\left(\eta_{13}, \phi_{13}\right),
$$

where

$$
\omega_{0}(\alpha)=\operatorname{diag}\left(e^{i \alpha_{1}}, e^{i \alpha_{2}}, e^{i \alpha_{3}}\right)
$$

with $\alpha_{3}=-\left(\alpha_{1}+\alpha_{2}\right)$ and, for example

$$
\omega_{12}\left(\eta_{12}, \phi_{12}\right)=\left[\begin{array}{ccc}
\cos \eta_{12} & e^{i \phi_{12}} \sin \eta_{12} & 0 \\
-e^{-i \phi_{12}} \sin \eta_{12} & \cos \eta_{12} & 0 \\
0 & 0 & 1
\end{array}\right] .
$$

Eq. (3.7) contains the eight parameters needed to characterize an arbitrary unitary unimodular matrix. From the standpoint of Eq. (3.6) it can be further simplified by using the freedom to rephase $\bar{e}_{L} \rightarrow \bar{e}_{L} \omega_{0}^{-1}(\alpha)$ without changing the free part of the charged lepton Lagrangian. On the other hand, the form of the mass terms in Eq. (3.1) shows that the neutrino fields can not be rephased. Thus a suitable minimal parameterization for $K$ in (3.6) is

$$
K=\omega_{23}\left(\eta_{23}, \phi_{23}\right) \omega_{12}\left(\eta_{12}, \phi_{12}\right) \omega_{13}\left(\eta_{13}, \phi_{13}\right)
$$

involving three "angles", $\eta_{a b}$ and three "phases", $\phi_{a b}$. Note the identity

$$
\omega_{0}(\alpha) \omega_{a b}\left(\eta_{a b}, \phi_{a b}\right) \omega_{0}^{-1}(\alpha)=\omega_{a b}\left(\eta_{a b}, \alpha_{a}+\phi_{a b}-\alpha_{b}\right)
$$

\footnotetext{
${ }^{\dagger}$ This is written out in detail in Eq (2) of 25]
} 
This identity may be used to transfer two of the phases $\phi_{a b}$ in Eq. (3.7) to a diagonal matrix on the right of $K$ as, for example,

$$
K=\omega_{23}\left(\eta_{23}, 0\right) \omega_{12}\left(\eta_{12}, 0\right) \omega_{13}\left(\eta_{13}, \phi_{13}\right) \omega_{0}^{-1}(\tau)
$$

where $\tau_{1}+\tau_{2}+\tau_{3}=0$, which may be used instead of Eq. (3.10).

We also need the formula for the amplitude of neutrino oscillation. For the case when a neutrino, produced by a charged lepton of type $a$, "oscillates" to make at time $t$, a charged lepton of type $b$, we have

$$
\operatorname{amp}(a \rightarrow b)=\sum_{\alpha} K_{a \alpha}^{*} K_{b \alpha} e^{-i E_{\alpha} t}
$$

where the sum goes over the neutrinos of definite mass, $m_{\alpha}$. Inserting the parameterization

Eq. (3.12) into Eq. (3.13) shows that the effect of the factor $\omega_{0}^{-1}(\tau)$ cancels out. Thus for ordinary oscillations, $K$ is parameterized by three angles and one CP violating phase as for the CKM quark mixing matrix. On the other hand, the two additional CP violating phases $\tau_{1}$ and $\tau_{2}$ show up if one considers neutrino-antineutrino oscillations [26] or neutrinoless double beta decay [27]. The formula for the probability, $P_{a b}$ is gotten by taking the squared magnitude of Eq. (3.13) and replacing the exponential factor $E_{\alpha} t$ by $\left(E+m_{\alpha}^{2} /(2 E)\right) L$, where $E$ is the neutrino energy and $L$ is the oscillation distance. For practical reasons it is very important to take account of the experimental uncertainties in $E$ and $L$. The simplest approximation [28] is to define $b=L /(4 E)$ and assume that one can smear $P_{a b}$ with a Gaussian distribution in $b . b_{0}$ is defined as the mean value and $\sigma_{b}$ as the standard deviation appropriate to the particular physical setup. Then we find for the smeared probability

$$
\begin{aligned}
\left\langle P_{a b}\right\rangle & =\delta_{a b}-2 \sum_{\alpha<\beta}\left[\operatorname{Re}\left(f_{\alpha \beta a b}\right)\left(1-\cos \left(2 b_{0} m_{\beta \alpha}^{2}\right) \exp \left(-2 \sigma_{b}^{2}\left(m_{\beta \alpha}^{2}\right)^{2}\right)\right)\right. \\
& \left.+\operatorname{Im}\left(f_{\alpha \beta a b}\right) \sin \left(2 b_{0} m_{\beta \alpha}^{2}\right) \exp \left(-2 \sigma_{b}^{2}\left(m_{\beta \alpha}^{2}\right)^{2}\right)\right]
\end{aligned}
$$

where $f_{\alpha \beta a b}=K_{a \alpha} K_{a \beta}^{*} K_{b \alpha}^{*} K_{b \beta}$ and $m_{\beta \alpha}^{2}=m_{\beta}^{2}-m_{\alpha}^{2}$. Notice that when $\operatorname{Im}\left(f_{\alpha \beta a b}\right)=0,\left\langle P_{a b}\right\rangle$ is independent of the sign of $m_{\beta \alpha}^{2}$.

\section{LEARNING ABOUT $M_{\nu}$ FROM EXPERIMENT}

Since $\operatorname{Tr}\left(M_{\nu}\right)=0$ provides only two real equations for 12 real parameters, it is clear that it has a relatively small amount of predictivity. In particular it cannot say much about the texture (e.g. possible zeroes) of $M_{\nu}$ which is suppposed to derive from a deeper theory than the standard model. On the other hand, we shall see that our Ansatz is complementary to 
the results which should emerge from analysis of neutrino oscillation experiments. Together, they should enable us to actually (with some conditions) reconstruct $M_{\nu}$.

First, in this section, we shall consider $M_{\nu}$ to be hermitian so that the argument in favor of $\operatorname{Tr}\left(M_{\nu}\right)=0$ presented in section II holds without any further assumptions. Since $M_{\nu}$ is symmetric it must be real. It can be brought to real diagonal form via a real rotation $R$ as $R^{T} M_{\nu} R$. However there is no guarantee that all eigenvalues of $M_{\nu}$ will be positive. We can make them all positive by rephasing the diagonal fields with negative eigenvalues by a factor $i$ (see Eqs. (3.1) and (3.2)). This means that the general diagonalizing matrix $U$ in Eq. (3.2) now takes the form

$$
U=R \omega
$$

where $\omega_{a b}=\delta_{a b} \eta_{b}^{\frac{1}{2}}$ with $\eta_{b}^{\frac{1}{2}}=1$ for a positive eigenvalue and $\eta_{b}^{\frac{1}{2}}=i$ for a negative eigenvalue. We notice that Eq. (4.1) is of the form Eq. (3.12) for which we already noticed that the factor $\omega$ cancels out in the neutrino oscillation formula Eq. (3.14). Furthermore only the square of the mass is relevant in Eq. (3.14). Thus we choose to work in this section with some negative masses and no factor $\omega$ in Eq. (4.1).

To avoid confusion, we remark that the factor $\omega$ in Eq. (4.1) does not introduce any CP violation in the theory [29] since $M_{\nu}$ is real in any event.

Now let us suppose that an experimental analysis of all neutrino oscillation experiments is made based on a formula like Eq. (3.14) (or one which treats the experimental uncertainties in a more sophisticated way). Furthermore assume that the CP violating phase $\phi_{13}$ in Eq. (3.12) is negligible. Then we should know the magnitudes of the squared neutrino mass differences

$$
\left(m_{2}\right)^{2}-\left(m_{1}\right)^{2}=A, \quad\left(m_{3}\right)^{2}-\left(m_{2}\right)^{2}=B
$$

where $A$ and $B$ can be either positive or negative. Then, assuming the leptonic theory to be CP conserving, our Ansatz would imply

$$
0=\operatorname{Tr}\left(M_{\nu}\right)=\operatorname{Tr}\left(R \hat{M} R^{T}\right)=m_{1}+m_{2}+m_{3}
$$

where Eq. (3.2) was used. Eqs. (4.2) and (4.3) comprise three equations for the three neutrino masses $m_{1}, m_{2}$ and $m_{3}$. We can solve to get:

$$
\begin{aligned}
\left(m_{1}\right)^{2} & =\frac{2}{3}\left[\sqrt{A^{2}+B^{2}+A B}-\left(A+\frac{B}{2}\right)\right], \\
m_{2} & =\frac{B-\left(m_{1}\right)^{2}}{2 m_{1}} \\
m_{3} & =-m_{1}-m_{2} .
\end{aligned}
$$


This leads to a limited number of solutions, depending on sign choices.

If we make the further assumption that the charged lepton mixing matrix $\Omega$ in Eq. (3.4) is approximately the unit matrix (this is expected to be a reasonable but not perfect approximation) we can identify $R \approx K$ which would be obtained from experiment. Then, using the masses found in Eq. (4.4), we could reconstruct $M_{\nu}$ as

$$
M_{\nu} \approx R \hat{M} R^{T}
$$

To proceed, we need only insert the experimental results for $A, B$ and $K$ in (4.4) and (4.5). Of course, it is presumably the task of the next decade to solidify the experimental determination of these quantities. We can, at the moment, only give a preliminary discussion. For this purpose we will use the results of a recent preliminary analysis of all neutrino experiments by Ohlsson and Snellman [30. These authors found, by a least square analysis, a best fit for (our notation) $|A|,|B|$ and the leptonic mixing matrix $K$. They used the formula Eq. (3.14) with a suitable choice of $b_{0}$ and $\sigma_{b}^{2}$ for each experiment. Furthermore, they made the simplifying assumption that $K$ is real. Finally they only searched for a fit in the range $10^{-4} \mathrm{eV}^{2} \leq|A| \leq 10^{-3} \mathrm{eV}^{2}, 0.2 \mathrm{eV}^{2} \leq|B| \leq 2 \mathrm{eV}^{2}$. This range corresponds to mass difference choices for which the MSW effect [31] for solar and atmospheric neutrinos is not expected to be important and so greatly simplifies the analysis. Thus there is no guarantee that the solution of [30] is unique. Altogether they fit sixteen different solar neutrino, atmospheric neutrino, accelerator and reactor experiments, including LSND. The best fit is:

$$
|A|=2.87 \times 10^{-4} \mathrm{eV}^{2}, \quad|B|=1.11 \mathrm{eV}^{2},
$$

for the squared mass differences and

$$
K_{\text {exp }}=\left[\begin{array}{ccc}
0.7052 & 0.7052 & 0.0732 \\
-0.6441 & 0.5940 & 0.4820 \\
0.2964 & -0.3871 & 0.8731
\end{array}\right]
$$

for the lepton mixing matrix $K$. As discussed above we will identify $K \approx R$ here, keeping $K$ real but allowing for negative masses. The best fit matrix $\mathrm{K}$ was obtained to be similar but not identical to the "bimaximal mixing" matrix [32].

With the best fit squared mass differences in Eq. (4.6), our model predicts, from the first of Eq. (4.4), eight different possibilities. These correspond to four different sign configurations for $A$ and $B$ times the two possible signs for $m_{1}$. However, only two of these eight are essentially different; these are 
type I : $m_{1}=0.6082 \mathrm{eV}, \quad m_{2}=0.6084 \mathrm{eV}, \quad m_{3}=-1.2166 \mathrm{eV}$

type II : $m_{1}=1.053701 \mathrm{eV}, \quad m_{2}=-1.053565 \mathrm{eV}, \quad m_{3}=-0.000136 \mathrm{eV}$

The other solutions correspond to interchanging whichever of $\left|m_{1}\right|$ and $\left|m_{2}\right|$ is greater (which has only a negligible effect since they are almost degenerate) or reversing the signs of all masses. Physically it is clear what is happening: the smallness of $|A|$ compared to $|B|$ in Eq. (4.2) forces $\left|m_{1}\right| \approx\left|m_{2}\right|$. Then we have either $m_{1} \approx m_{2}$ with, using the constraint Eq. (4.3), $m_{3} \approx-2 m_{1}$ or $m_{1} \approx-m_{2}$ with $m_{3}$ very small.

Since we have assumed the neutrinos to be of Majorana type for our plausibility argument in section II, their interactions will violate lepton number. Then they should mediate neutrinoless double beta decay $\left(\beta \beta_{0 \nu}\right)$ [33]. Such a process has not yet been observed and an upper bound has been set for the relevant quantity

$$
\left\langle m_{\nu}\right\rangle \equiv\left|\sum_{\alpha=1}^{3}\left(K_{1 \alpha}\right)^{2} m_{\alpha}\right| .
$$

The best upper bound at present is [34] $\left\langle m_{\nu}\right\rangle \leq 0.2-0.6 \mathrm{eV}$, reflecting some uncertainty in the estimation of the needed nuclear matrix elements.

Substituting the best fit for the matrix K from Eq. (4.7) together with our results in Eqs. (4.8) and (4.9) into Eq. (4.10) yields predictions for the two cases:

$$
\begin{aligned}
\text { type I : } & \left\langle m_{\nu}\right\rangle=0.60 \mathrm{eV}, \\
\text { type II : } & \left\langle m_{\nu}\right\rangle=6.7 \times 10^{-5} \mathrm{eV} .
\end{aligned}
$$

Both solutions seem to be acceptable, the type I case marginally but the type II case definitely. Note that the small value for $\left\langle m_{\nu}\right\rangle$ in the type II case is due to the best fit prediction [30] $K_{11}=K_{12}$ and also to the fact that $m_{2}$ is negative. The same value would clearly result if we made $m_{2}$ positive and set $K_{12}=0.7052 i$ as discussed around Eq. (4.1) above.

Finally, let us reconstruct the underlying neutrino mass matrices for each of the two cases. We use Eq. (4.5) based on the assumption that $M_{\nu}$ is real and also our ansatz to find (in units of $\mathrm{eV}$ ):

$$
\begin{aligned}
\text { type I : } \quad M_{\nu}=M_{\nu}^{T} \approx\left[\begin{array}{ccc}
0.5985 & -0.0643 & -0.1167 \\
-0.0643 & 0.1843 & -0.7680 \\
-0.1167 & -0.7680 & -0.7828
\end{array}\right], \\
\text { type II : } \quad M_{\nu}=M_{\nu}^{T} \approx\left[\begin{array}{ccc}
6.7 \times 10^{-5} & -0.9199 & 0.5078 \\
-0.9199 & 0.0654 & 0.0410 \\
0.5078 & 0.0410 & -0.0654
\end{array}\right] .
\end{aligned}
$$


The type I matrix does not have an excellent candidate for a "texture" zero. However the small value of $\left(M_{\nu}\right)_{11}$ in the type II case is certainly suggestive. These matrices lead to neutrino masses and a mixing matrix which give a best fit to all present data. It will be interesting to see if either of them hold up in the future.

Incidentally, on comparing Eqs. (4.13) and (4.14) it is amusing to observe the large difference in two mass matrices "generated" in the same way except with respect to how $\operatorname{Tr}\left(M_{\nu}\right)=0$ is satisfied.

\section{CASE OF COMPLEX $M_{\nu}$}

It seems interesting to also investigate the Ansatz $\operatorname{Tr}\left(M_{\nu}\right)=0$ when $M_{\nu}$ is no longer restricted to be real. This also raises the problem of constructing the unitary diagonalizing matrix $U$ in Eq.(3.2), in terms of the experimentally measured lepton mixing matrix $K_{\text {exp }}$. For simplicity, as before, we will make the approximation that the charged lepton diagonalizing matrix $\Omega$ is the unit matrix.

Apart from an overall (conventional) phase we may write

$$
U=\omega_{0}(\sigma) K_{\exp } \omega_{0}^{-1}(\tau)
$$

where the 2-parameter quantity $\omega_{0}$ was defined in Eq. (3.8). Since $K_{\text {exp }}$ has four parameters $U$ in Eq. (5.1) is described by eight parameters. As mentioned before, the two parameters in $\omega_{0}^{-1}(\tau)$ are not measurable in neutrino oscillation experiments but show up when one considers $(\beta \beta)_{o \nu}$. The two parameters in $\omega_{0}(\sigma)$ may be eliminated, for experimental purposes, by rephasing the charged leptons. However, for the theoretical purpose of reconstructing the underlying neutrino mass matrix $M_{\nu}$, their existence cannot be ruled out. (They also do not contribute to $(\beta \beta)_{0 \nu}$.)

For the purpose of relating the Ansatz on $M_{\nu}$ to the physical neutrino masses in $\hat{M}$, we note

$$
\operatorname{Tr}\left(M_{\nu}\right)=\operatorname{Tr}\left(K_{\text {exp }}^{-1} \omega_{0}^{-1}(2 \sigma) K_{\text {exp }}^{*} \omega_{0}(2 \tau) \hat{M}\right)
$$

For further simplicity of the analysis we adopt the special case $\omega_{0}(2 \sigma)=1$ and also identify $K_{\text {exp }}$ with the real best fit in Eq. (4.7); our Ansatz now reads

$$
0=\operatorname{Tr}\left(M_{\nu}\right)=\operatorname{Tr}\left(\omega_{0}(2 \tau) \hat{M}\right)
$$

With the redefinitions $\beta_{1}=4 \tau_{1}+2 \tau_{2}$ and $\beta_{2}=2 \tau_{1}+4 \tau_{2}$, Eq. (5.3) becomes 


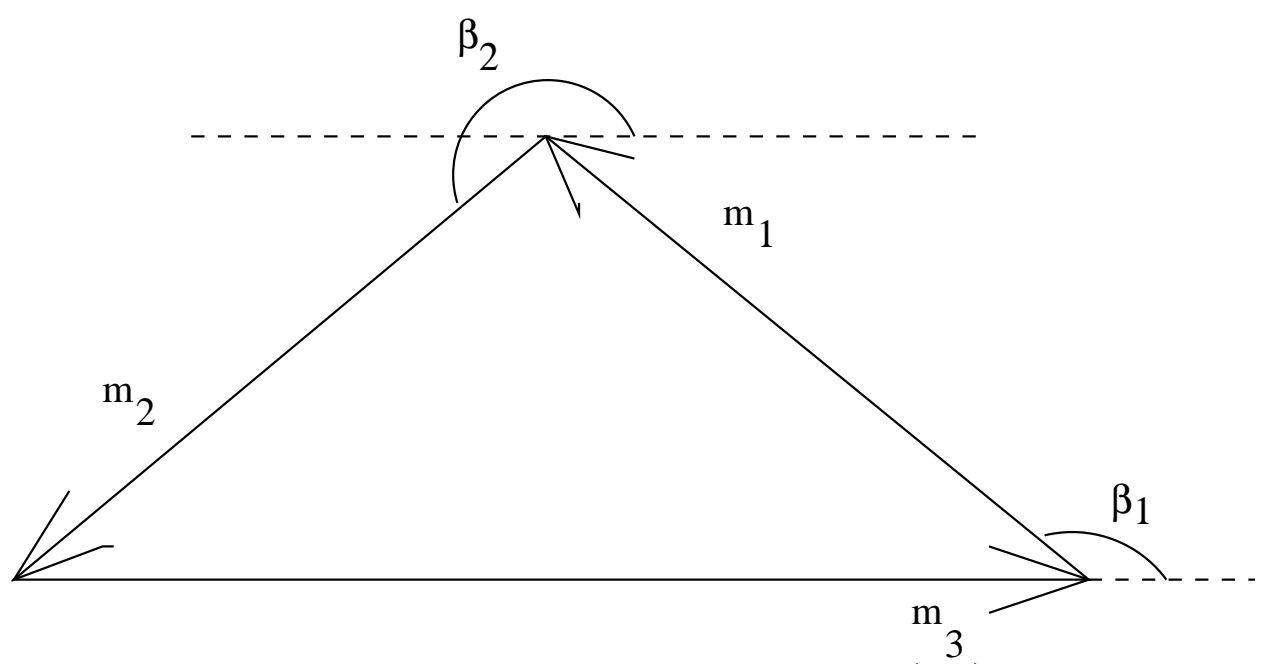

FIG. 1. Geometrical picture of Eq. (5.4).

$$
e^{i \beta_{1}} m_{1}+e^{i \beta_{2}} m_{2}+m_{3}=0
$$

This may be conveniently visualized as the vector triangle shown in Fig. 1.

Combining Eqs. (5.4) and (4.2) gives four real equations for the five unknown quantities $\left(m_{1}, m_{2}, m_{3}, \beta_{1}, \beta_{2}\right)$. Thus we have (for each set of $(A, B)$ sign choices) a one parameter family of solutions. It is convenient to choose this parameter to be $m_{3}$. Then $m_{1}$ and $m_{2}$ may be found from the equations (4.2), provided that solutions exist. In this way all three sides of the triangle in Fig.1 1 are determined. The angles may finally be found as

$$
\begin{aligned}
\cos \beta_{2} & =\frac{m_{1}^{2}-m_{2}^{2}-m_{3}^{2}}{2 m_{2} m_{3}} \\
\sin \beta_{1} & =-\frac{m_{2}}{m_{1}} \sin \beta_{2} .
\end{aligned}
$$

We also need to investigate the constraint arising from the non-observation of $(\beta \beta)_{0 \nu}$. Eq. (4.10) now becomes, with Eq. (5.1) as the mixing matrix

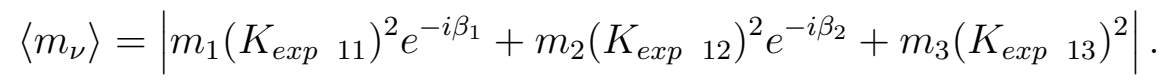

Using the Ansatz constraint Eq. (5.4), Eq. (5.6) may be rewritten as

$$
\left\langle m_{\nu}\right\rangle=\left|\left[\left(\begin{array}{ll}
K_{\exp } & 12
\end{array}\right)^{2}-\left(K_{\exp } 11\right)^{2}\right] m_{2} e^{-i \beta_{2}}+\left[\left(\begin{array}{ll}
K_{\exp } & 13
\end{array}\right)^{2}-\left(\begin{array}{ll}
K_{\exp } & 11
\end{array}\right)^{2}\right] m_{3}\right| .
$$

This form is very convenient when identifying $K_{\text {exp }}$ with the best fit solution in Eq. (4.7). In the present context such an identification corresponds to $\mathrm{CP}$ violation for the $(\beta \beta)_{0 \nu}$ process but not for usual neutrino oscillations. Since the (11) and (12) matrix elements are equal in Eq. (4.7) we find the simple result 


$$
\left\langle m_{\nu}\right\rangle=0.49 m_{3}
$$

Thus if the upper bound on $\left\langle m_{\nu}\right\rangle$ is conservatively identified as in the $0.2-0.6 \mathrm{eV}$ range, we should have in this case

$$
m_{3} \leq 0.41-1.22 \mathrm{eV}
$$

In the present complex case there is a continuum of possible solutions labelled by those values of $m_{3}$ satisfying Eq. (5.9), rather than just the two possibilities found in Eq. (4.8) and Eq. (4.9). Actually, the continuum separates roughly into two classes similar to either Eq. (4.8) or Eq. (4.9). In the generalized type I class, $m_{3}$ is of the order $B=1.11 \mathrm{eV}$ while $m_{1}$ and $m_{2}$ are related to nearly oppositely directed vectors in Fig. 1 and are also of the order $B$. In the generalized type II class, $B$ is negative; $m_{1}$ and $m_{2}$ correspond to vectors of order $|B|$ which are oppositely directed to each other, while $m_{3}$ ranges from very small to order $|B|$.

Given the bound Eq. (5.9) from the non-observation of $(\beta \beta)_{o \nu}$, there are important limitations on the allowed $m_{3}$ values for type I solutions. In this case $B$ is positive so the equation

$$
\left(m_{2}\right)^{2}=\left(m_{3}\right)^{2}-B=\left(m_{3}\right)^{2}-(1.11 \mathrm{eV})^{2}
$$

will only allow solutions for $m_{3}>1.11 \mathrm{eV}$. This range is barely compatible with Eq. (5.9). Thus the type II case where $B<0$ and $m_{1} \approx m_{2}>m_{3}$ seems most probable.

As an example of a solution for complex $M_{\nu}$, consider choosing $m_{3}=0.2 \mathrm{eV}$. Then a solution is obtained with (compare with Fig. 1)

$$
m_{1} \approx m_{2} \approx 1.128 \mathrm{eV}, m_{3}=0.2 \mathrm{eV}, \beta_{1} \approx 95.1^{\circ}, \beta_{2} \approx 264.9^{\circ}
$$

The matrix $U$, which diagonalizes $M_{\nu}$ is obtained from Eq. (5.1), with the approximation $\omega_{0}(\sigma)=1$, and with now:

$$
\omega_{0}^{-1}(\tau)=\operatorname{diag}(0.976+0.216 i, 0.301-0.953 i, 0.500+0.866 i)
$$

This factor introduces $\mathrm{CP}$ violation in the $(\beta \beta)_{0 \nu}$ process but not in ordinary neutrino oscillations.

Finally the underlying neutrino mass matrix, $U \hat{M} U^{T}$ is "reconstructed" as (in units of $\mathrm{eV})$ :

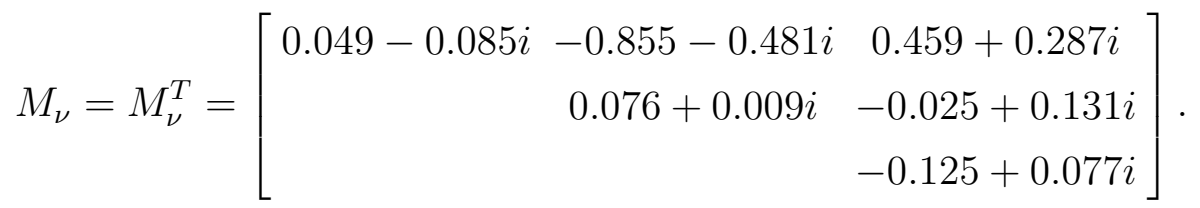


This is structurally similar to the real type II solution displayed in Eq. (4.14), although the suppression of the (11) element is not so pronounced. Notice that $m_{3}$ is considerably smaller than the almost degenerate pair $m_{1}$ and $m_{2}$. Furthermore $m_{1}$ and $m_{2}$ are large enough to possibly play some role in astrophysics.

\section{SUMMARY AND DISCUSSION}

We investigated the Ansatz $\operatorname{Tr}\left(M_{\nu}\right)=0$ for the underlying (pre-diagonal) three generation neutrino mass matrix. It was motivated by noting that in an $\mathrm{SO}(10)$ grand unified model where $M_{\nu}$ was taken to be real (CP conserving), it corresponds to the well known unification of $\mathrm{b}$ quark and $\tau$ lepton masses. While not very predictive by itself it yields information complementary to what would be gotten from a complete three flavor analysis of all lepton number conserving neutrino oscillation experiments. Specifically from the specification of the magnitudes of two neutrino squared mass differences and also of the leptonic mixing matrix we can, with some assumptions, find the neutrino masses themselves and "reconstruct" $M_{\nu}$. This determination can be sharpened by consideration of the constraints imposed by non-observation of neutrinoless double beta decay.

For the purpose of testing our Ansatz we employed the results of a reasonable best fit to all present neutrino experiments (including LSND) by Ohlsson and Snellmann [30. This fit will inevitably be improved in the next few years as new experiments are completed. They were able to fit the data without assuming any CP violation. This agrees with assuming $M_{\nu}$ to be real. We found two essentially different solutions in that case. The first features two neutrinos having approximately equal mass $0.608 \mathrm{eV}$ and a third neutrino of mass 1.217 $\mathrm{eV}$. This solution is on the borderline of being ruled out by non-observation of $\left(\beta \beta_{0 \nu}\right)$. The second solution has two neutrinos with approximately degenerate mass $1.054 \mathrm{eV}$ and a third neutrino with a mass $1.36 \times 10^{-4} \mathrm{eV}$. This solution is very safe from being ruled out by $\left(\beta \beta_{0 \nu}\right)$ experiments. It also features a reconstructed $M_{\nu}$ which has an extremely small (11) element. Note that, for both solutions, even though $M_{\nu}$ is real there are some (CP conserving) factors of $i$ in the mixing matrix when all masses are taken to be positive. Alternatively one may have no $i$ 's in the mixing matrix while allowing some masses to be negative. The latter form is useful for seeing intuitively how $\operatorname{Tr}\left(M_{\nu}\right)=0$ is possible.

The case of matching the above best fit data to a complex $M_{\nu}$ was also considered. In this situation there are $\mathrm{CP}$ violating phases in the lepton mixing matrix which affect the $(\beta \beta)_{0 \nu}$ process but do not affect ordinary total lepton number conserving neutrino oscillations. Such phases could also be measurable in principle with the observation of a decay like $\tau^{-} \rightarrow$ 
$\pi^{-} \pi^{-} \mu^{+}$. The case of complex $M_{\nu}$ allows a larger number of solutions. With a simplifying assumption there is a one parameter family of allowed neutrino mass sets. Roughly, these fall into one of the two types already encountered for real $M_{\nu}$.

A question of some interest is whether the neutrinos are massive enough to play a role in cosmology. The relevant criterion [35] for this to occur is usually stated as $\sum_{a} m_{a}>6 \mathrm{eV}$. For the type II solutions with complex $M_{\nu}$ we have found the largest mass sum to be about $4.5 \mathrm{eV}$ corresponding to $m_{1} \approx m_{2}=1.62 \mathrm{eV}$ and $m_{3} \approx 1.22 \mathrm{eV}$. However this is on the very border of acceptability for non observation of $(\beta \beta)_{0 \nu}$.

Future best fits to the neutrino oscillation data can easily be accommodated in the present framework. Of course, the predictions for neutrino masses and mixings will depend on this input.

\section{ACKNOWLEDGMENTS}

We would like to thank H. Benaoum for useful comments on the manuscript. This work has been supported in part by the US DOE under contract DE-FG-02-85ER 40231.

[1] Super-Kamiokande Collaboration, Y. Fukuda et al., Phys. Rev. Lett. 81, 1562 (1998). SuperKamiokande and Kamiokande Collaborations, T. Kajita, in Proceedings of the XVIIIth International Conference on Neutrino Physics and Astrophysics (Neutrino '98), Takayama, Japan, 1998 [Nucl. Phys. B (Proc. Suppl.) 77, 123 (1999)].

[2] Soudan Collaboration, W.W.M. Allison et al., Phys. Lett. B 391, 491 (1997);

[3] MACRO Collaboration, M. Ambrosio et al., Phys. Lett. B 434, 451 (1998).

[4] Kamiokande Collaboration, S. Hatakeyama et al., Phys. Rev. Lett. 81, 2016 (1998).

[5] IMB Collaboration, R. Becker-Szendy et al., Phys. Rev. D 46, 3720 (1992).

[6] SAGE Collaboration, J.N. Abdurashitob et al. Phys. Rev. Lett. 77, 4708 (1996).

[7] GALlEX Collaboration, P. Anselmann et al., Phys. Lett. B 342, 440 (1995); W. Hampel et al., Phys. Lett. B 388, 384 (1996).

[8] Homestake Collaboration, B.T. Cleveland et al., Astrophys. J. 496, 505 (1998). 
[9] Super-Kamiokande Collaboration, Y. Fukuda et al., Phys. Rev. Lett. 81, 1158 (1998); SuperKamiokande Collaboration, Y. Fukuda et al., ibid. 81, 4279 (1998).

[10] Kamiokande Collaboration, Y. Fukuda et al., Phys. Rev. Lett. 77, 1683 (1996);

[11] CHOOZ Collaboration, M. Apollonio et al., Phys. Lett. B 420, 397 (1998); CHOOZ Collaboration, C. Bemporad, in Proceedings of the XVIIIth International Conference on Neutrino Physics and Astrophysics (Neutrino '98), Nucl. Phys. B (Proc. Suppl.) 77159 (1999);

[12] Bugey Collaboration, B. Achkar et al., Nucl. Phys. B434, 503 (1995).

[13] KARMEN Collaboration, B. Armbruster et al., Phys. Rev. C 57, 3414 (1998); Phys. Lett. B 423, 15 (1998); K Eitel et al., Nucl. Phys. B (Proc. Suppl.) 77, 212 (1999).

[14] NOMAD Collaboration, J. Altegoer et al., Phys. Lett. B 431, 219 (1998).

[15] CHORUS Collaboration, P. Migliozzi, talk given at the XXIXth International Conference on High-Energy Physics (ICHEP 98), hep-ex/9807024.

[16] LSND Collaboration, C. Athanassopoulos et al., Phys. Rev. Lett. 77, 3082 (1995); LSND Collaboration, C. Athanassopoulos et al., ibid. 81, 1774 (1998).

[17] E. K. Akhmedov, hep-ph/0001264; J. N. Bahcall, Nucl. Phy. Proc. Suppl. 48, 309(1996); S. Bilenky, hep-ph/990246; P. Ficher, B. Kayser and K. S. McFarland, Anual Review of Nuclear and Particle Physics, 49 (1999); D. R. O. Morrison, Part. World 3, 30(1992); A. Rubbia, Acta. Phys. Polon. B 30, 2351(1999); A. Y. Smirnov, hep-ph/9901208; J. W. F. Valle, hepph/9911224, hep-ph/9906539; J. D. Vergados, Phys. Rep. 133 (1986).

[18] C. H. Albright and S. M. Barr, Phys. lett. B461, 218(1999); V. Barger, S. Pakvasa, T. J. Weiler and K.Whisnant, Phys. Lett. B437, 107(1998); H. B. Benaoum and S. Nasri; Phys. Rev. D60, 113003(1999); M. Jezabek and Y. Sumino, Phys. Lett. B457, 139(1999); S. K. Kang and C. S. Kim, Phys. Rev. D59, 091302(1999); R. N. Mohapatra and S. Nussinov, Phys. Rev. D60, 013002(1999), R. N. Mohapatra, A. Perez-Lorenzano and C .A. de Sousa Pires, Phys. Lett. B474, 355(2000); H. Georgi and S. L. Glashow, Phys. Rev. D61, 097301 (2000).

[19] H. Georgi, Particle and Fields (1974), ed. C. E. Carlson (AIP, New York, 1975) p 575; H. Fritzch and P. Minkowski, Ann. Phys. (NY) 93, 193 (1975); Nucl. Phys. B103, 61 (1976).

[20] T. Yanagida, Proc. of the Workshop on Unified Theory and Baryon Number in the Universe, ed. by O. Sawada and A. Sugamato (KEK Report 79-18,1979), p 95; M. Gell-Mann, P. Ramond and R. Slansky in Supergravity, eds P. van Niewenhuizen and D. Z. Freedman (North Holland, 
1979); R. N. Mohapatra and G. Senjanovic, Phys. Rev. Lett. 44, 912 (1980).

[21] See for example K. Matsuda, T. Fukuyama and H. Nishiura, Phys. Rev. D 61, 053001(2000); A. Bottino, C. W. Kim, H. Nishiura and W. K. Tse, Phys. Rev. D 34, 862 (1986); K. S. Babu and R. N. Mohapatra, Phys. Rev. Letts. 70, 2845 (1993).

[22] A. J. Buras, J. Ellis, M. K. Gaillard, and D. V. Nanopoulos, Nucl. Phys. B135, 66(1978).

[23] J. Schechter and J. W. F. Valle, Phys. Rev. D22, 2227(1980).

[24] R. Johnson, S. Ranfone and J. Schechter, Phys. Lett. B 179, 355 (1986); Phys. Rev. D 35, $282(1987)$.

[25] M. Gronau and J. Schechter, Phys. Rev. Lett.54, 385(1985). Note that the second minus sign in the (12) element of Eq (2) should be plus.

[26] J. Schechter and J. W. F. Valle, Phys. Rev. D 23, 1666(1981).

[27] For a detailed review see M. Doi, T. Kotani and E. Takasugi, Prog. Theor. Phys. Supplement No. 83.

[28] Review of Particle Physics, Europ. Phys. Journal 3, 1(1999). See p320.

[29] L. Wolfenstein, Phys. Lett. B 107, 77 (1981).

[30] T. Ohlsson and H. Snellman, Phys. Rev. D. 60, 093007 (1999).

[31] S. P. Mikhyev and A. Y. Smirnov, Nuovo Cimento 9C, 17 (1986); L. Wolfenstein, Phys. Rev. D 17, 2369 (1978).

[32] V. Barger et al, ref. 17 above.

[33] S. M. Bilenky, C. Giunti and W. Grimus Prog. Part. Nucl. Phys. 431 (1999).

[34] Heidelberg-Moscow Collaboration, L. Baudis et al., Phys. Rev. Lett. 83, 41 (1999); H. V. Klapdor-Kleingrothaus, Int. J. Mod. Phys. 133953 (1998).

[35] G. Steigman, Nucl. Phys. Proc. Suppl. 31, 343 (1993); D. N. Schramm, Nucl. Phys. Proc. Suppl. 38, 349 (1995); G. Gelmini, hep-ph/9904369. 